\title{
Lin28A induces energetic switching to glycolytic metabolism in human embryonic kidney cells
}

\author{
Craig K. Docherty ${ }^{1}$, Ian P. Salt ${ }^{1}$ and John R. Mercer ${ }^{1,2^{*}}$
}

\begin{abstract}
Background: Loss of a cell's capacity to generate sufficient energy for cellular functions is a key hallmark of the ageing process and ultimately leads to a variety of important age-related pathologies such as cancer, Parkinson's disease and atherosclerosis. Regenerative medicine has sought to reverse these pathologies by reprogramming somatic cells to a more juvenile energetic state using a variety of stem cell factors. One of these factors, Lin28, is considered a candidate for modification in the reprogramming of cellular energetics to ameliorate the ageing process while retaining cell phenotype.

Results: Over-expression of Lin28A resulted in key changes to cellular metabolism not observed in wild-type controls. Extracellular pH flux analysis indicated that Lin28A over expression significantly increased the rate of glycolysis, whilst high resolution oxygen respirometry demonstrated a reduced oxygen consumption. Western blot and real-time PCR analysis identified Hexokinase II as one of the key modulators of glycolysis in these cells which was further confirmed by increased glucose transport. A metabolic switching effect was further emphasised by Western blot analysis where the oxygen consuming mitochondrial complex IV was significantly reduced after Lin28A over expression.
\end{abstract}

Conclusions: Results from this study confirm that Lin28A expression promotes metabolic switching to a phenotype that relies predominantly on glycolysis as an energy source, while compromising oxidative phosphorylation. Mechanisms to augment regulated Lin28A in age related pathologies that are characterised by mitochondria dysfunction or in differentiated and aged post-mitotic cells is the future goal of this work.

Keywords: Lin28, Cellular metabolism, Glycolysis, Hexokinase II, Reprogramming

\section{Background}

Regulation of cellular metabolism holds great potential to intervene in the progression of human disease and ageing [1]. Research suggests that loss of a cell's energetic capacity during ageing and age related pathologies can be causative and therefore amenable to intervention [2-4]. Regenerative medicine has identified stem cell factors capable of fundamentally reorganising cellular metabolism and cell phenotype which may further be able to reprogram metabolism within the context of cancer, cardiovascular disease and kidney disease [5-7]. However, to

\footnotetext{
* Correspondence: John.Mercer@glasgow.ac.uk

${ }^{1}$ Institute of Cardiovascular and Medical Sciences, College of Medical, Veterinary and Life Sciences, University of Glasgow, Glasgow G12 8TA, Scotland, UK ${ }^{2}$ BHF Glasgow Cardiovascular Research Centre, University of Glasgow, Glasgow G12 8TA, Scotland, UK
}

provide a new therapeutic avenue in differentiated somatic cell types their mechanism of action needs to be clarified.

Lin28 is an RNA binding protein that positively regulates embryogenesis timing and progenitor self-renewal [8]. Recognised as one of the key pluripotency markers it exists as two conserved paralogs, Lin28A and Lin28B, which are highly expressed during embryogenesis, where it promotes cell growth and maintenance of a more juvenile energetic phenotype [9]. Both homologs repress the Let-7 (lethal-7) family of regulatory microRNAs (miRNA) [10, 11] required for terminal cell differentiation by inhibiting the drosha and dicer microprocessor complexes required for the production of mature miRNA [1]. Most mature cells observe down regulation of Lin28 that occurs during differentiation with a concurrent 
increase in expression of mature Let7 miRNAs to maintain terminal cell fate [10].

Metabolomic profiling confirms promotion of an embryonic like bio-energetic state that leads to an enhanced metabolism from glycolysis [1, 10, 12]. Enhanced glucose metabolism is a key hallmark of functional Lin28 within these cells. Indeed, Lin28 over expression in both in vitro and in vivo models demonstrates increased glucose metabolism via increased glucose uptake and an increase in the glycolytic enzyme pyruvate dehydrogenase kinase 1 PDK1 [5]. Over-expression of Lin28 also results in increased tissue repair and an improved glucose tolerance [13]. However, unregulated Lin28 expression is also implicated in human malignancies through increases in glycolytic metabolism and cellular proliferation [13-15] intimating that unregulated Lin 28 may be oncogenic. Studies on human embryonic stem cells have also suggested that the Lin28/Let 7 axis interacts with multiple mitochondrial enzymes suggesting further effects on oxidative phosphorylation [16]. Therefore, modification of Lin28 expression may have multiple roles in tissue regeneration and reprogramming metabolism.

Although many in vitro studies have elucidated the role of Lin28 in glycolysis, the exact role Lin28 plays in regulating oxidative phosphorylation is unknown. This study outlines key effects Lin28 has on mitochondrial activity and demonstrates further specific effects Lin 28 has on glycolysis.

\section{Methods}

\section{Cell culture}

Cells were maintained in high glucose ( $4.5 \mathrm{~g} / \mathrm{L})$ Dulbecco's Modified Eagle's Medium (DMEM) containing $15 \%$ (v/v) foetal bovine serum, $2 \mathrm{mM}$ L-glutamine, $1 \mathrm{mM}$ pyruvate, $100 \mathrm{U} / \mathrm{ml}$ penicillin and $100 \mathrm{mg} / \mathrm{ml}$ streptomycin. Lin $28 \mathrm{~A}$ cells were supplemented with $10 \mu \mathrm{g} / \mathrm{ml}$ of the selection reagent Basticidin-S hydrochloride (Sigma). Lin28A overexpressing human embryonic kidney (HEK) 293 cells were obtained from Amsbio, UK. Non-transfected HEK293 cells were obtained from Professor Andrew Baker (University of Edinburgh).

\section{Quantitative real-time PCR}

For gene expression experiments cells were grown in six-well plates until they reached $90-100 \%$ confluency. QIAzol lysis reagent was added to wells and cells were scraped into RNAse free micro-centrifuge tubes and stored at $-80{ }^{\circ} \mathrm{C}$. RNA species were then extracted with the miRNeasy ${ }^{\circ}$ mini kit (Qiagen), quantified using a nanodrop spectrophotometer and normalised. RNA was then reverse transcribed to cDNA and miRNA using a TaqMan $^{\circ}$ reverse transcription kit and an miRNA reverse transcription Kit, respectively (Applied Biosystems). For miRNA, the amplification step was performed using specific TaqMan miRNA probes. Real-time PCR was performed using the Applied Biosystems 7900 HT real-time PCR system following the manufacturer's instructions. Specific TaqMan ${ }^{\circ}$ primer-probes were purchased from ThermoFisher (Additional file 1: Table S1) and analysed using the delta CT method.

\section{Western blotting}

Cells were lysed in $1 \%(\mathrm{w} / \mathrm{v})$ lauryl maltoside detergent (Abcam) in PBS and sonicated. A total of $20 \mu \mathrm{g}$ of protein lysates was fractionated on 4-12\% gradient polyacrylamide gels and transferred to nitrocellulose membranes (Amersham). Membranes were then blocked in a 1:1 mix of SEA block (Thermo-Fisher) and Trisbuffered saline containing $0.1 \%(\mathrm{v} / \mathrm{v})$ tween 20 (TBST). Membranes were incubated overnight at $4{ }^{\circ} \mathrm{C}$ with the relevant primary antibody. Membranes were then washed in TBS/T and incubated with fluorescent secondary antibody (1:15000) for 2 hours. Membranes were then transferred to the LI-COR Odyssey-Sa infrared imaging system for visualisation and quantification. Densitometric analysis was then performed on LI-COR image studio light (version 5.2). All primary antibody dilutions are outlined in Additional file 1: Table S2.

\section{Extracellular flux analysis}

Extracellular acidification rate (ECAR) was evaluated using the Seahorse XF24 analyser (Seahorse Bioscience, MA, USA). Briefly, $5 \times 10^{4}$ cells were seeded in 24-well seahorse plates 24 hours prior to the experimental run. On the day of the experiment media were changed to glucose and sodium pyruvate-free XF assay media and transferred to the XF24 analyser (\#102365-100; Seahorse Bioscience, MA, USA). ECAR was determined after the sequential addition of D-glucose (10 $\mathrm{mM}$ final), oligomycin $(1 \mu \mathrm{M})$ and 2-deoxyglucose (2-DG; $100 \mathrm{mM})$. All compounds were purchased from Sigma, USA.

\section{High resolution oxygen respirometry}

Cells were trypsinized and diluted in MiR05 respiration buffer $(0.5 \mathrm{mM}$ EGTA, $3 \mathrm{mM} \mathrm{MgCl} 2,60 \mathrm{mM}$ potassium

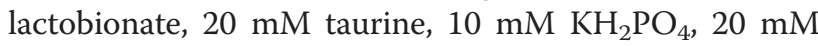
HEPES- $\mathrm{KOH} \mathrm{pH}$ 7.1, $110 \mathrm{mM}$ sucrose, and $1 \mathrm{~g} / \mathrm{L}$ fatty acid free BSA) (Oroboros, Innsbruck, Austria) to $0.5 \times$ $10^{6}$ per $\mathrm{ml}$ and $2 \mathrm{ml}$ of cells were transferred to the $\mathrm{O} 2 \mathrm{~K}$ Oroborus respirometer (Oroboros, Innsbruck, Austria). Oxygen consumption rate (OCR) was determined after the sequential addition of oligomycin $(1 \mu \mathrm{M})$, carbonyl cyanide m-chlorophenylhydrazone (CCCP; 1-3 $\mu \mathrm{M})$, rotenone $(1 \mu \mathrm{M})$ and antimycin $(1 \mu \mathrm{M})$.

\section{Cell proliferation analysis}

Cells were transferred to 12 -well plates at a seeding density of $1 \times 10^{4}$ cells $/ \mathrm{ml}$. Cell numbers from three 
wells were then counted every 24 hours over a 4-day period using a Hemacytometer.

\section{Glucose transport assay}

2-[ $\left.{ }^{3} \mathrm{H}\right]$ Deoxy-D-glucose transport was measured in cells grown on12-well plates. Briefly, cells were incubated in phosphate-buffered Krebs solution (KRP buffer; $128 \mathrm{mM}$ $\mathrm{NaCl}, 4.7 \mathrm{mM} \mathrm{KCl}, 5 \mathrm{mM} \mathrm{NaH} \mathrm{PO}_{4}, 1.2 \mathrm{mM} \mathrm{MgSO}$, $2.5 \mathrm{mM} \mathrm{CaCl} 2,5 \mathrm{mM}$ glucose and $0.1 \% \mathrm{BSA}$ ) for 30 mins at $37{ }^{\circ} \mathrm{C}$ then transferred to glucose-free KRP for a further 30 mins. Glucose transport was initiated by addition of 2- $\left[{ }^{3} \mathrm{H}\right]$ deoxy-D-glucose (final concentration $25 \mu \mathrm{mol} / \mathrm{L}$ and $1 \mu \mathrm{Ci} / \mathrm{ml}$ ) to each well. The mixture was then incubated for $5 \mathrm{~min}$. Non-specific association of radioactivity was determined by prior addition of cytochalasin B $(10 \mu \mathrm{mol} / \mathrm{l})$. Cell culture plates were then immersed in ice-cold PBS and allowed to dry before addition of $1 \%(\mathrm{v} / \mathrm{v})$ Triton X-100. Samples were added to scintillation vials 24 hours later and radioactivity was measured using a Beckman LS6500 scintillation counter.

\section{Data analysis}

All data are presented as mean \pm standard error of the mean (SEM) where appropriate. P values were calculated using student's unpaired t-tests (two-tailed distribution). Statistical significance was displayed as $\mathrm{P}<0.05$ (one star), $\mathrm{P}<0.01$ (two stars) or $\mathrm{P}<0.001$ (three stars). All analysis was performed on Microsoft Office Excel 2007 or Graphpad Prism (v 5). All graphs were produced on Graphpad Prism (v 5).

\section{Results}

\section{Characterisation of Lin28 in HEK293 cells}

To identify the effect of over-expression of Lin28 in our cell lines, we first examined mRNA expression using q-PCR (Taqman) (Fig. 1a). Increased Lin28A mRNA resulted in a concomitant downregulation of the dominant Let-7 miRNA transcripts let $7 \mathrm{a}(\mathrm{P}<0.001)$, c $(\mathrm{P}<0.001)$ and $g(P<0.05)($ Fig. $1 b)$. To examine the functional effects of Lin28 over-expression, Lin28 protein levels were also assessed (Fig. 1c). HEK293 cells overexpressing Lin28 exhibited significantly up-regulated protein expression compared to wild-type controls $(\mathrm{P}<0.001)$ (Fig. 1d).

\section{Lin28 over-expression induces glycolytic 'switching'}

Over-expression of Lin28 significantly increased the maximum glycolytic rate within HEK293 cells (Fig. 2a). The extracellular acidification rate (ECAR) is a measure of milli-pH change attributed to accumulation of glycolytic pyruvate acid and is a measure of glycolytic rate (Seahorse Technologies ${ }^{\circ}$ ). We found that Lin28 enhanced glycolysis $(\mathrm{P}<0.001)$, glycolytic capacity $(\mathrm{P}<0.001)$ and glycolytic
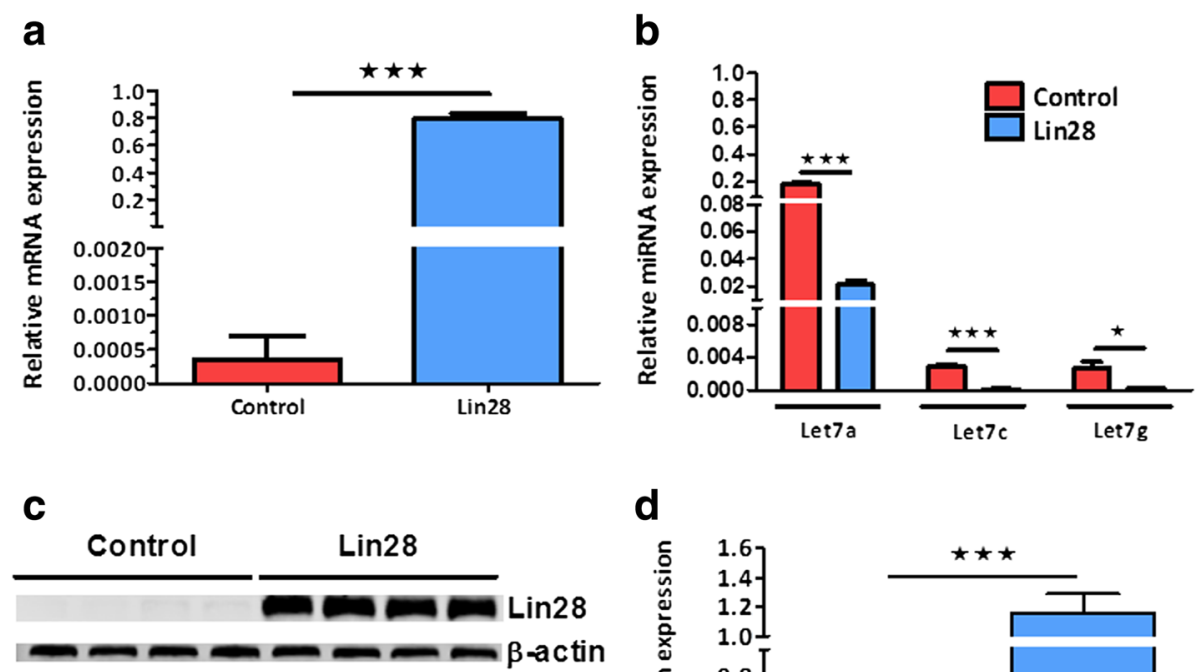

d

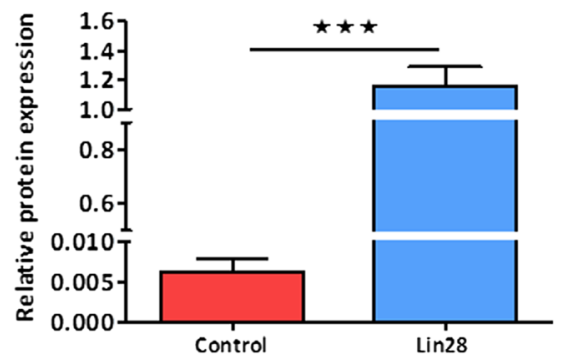

Fig. 1 Characterisation of Lin28A over-expression in HEK 293 cells. Real-time PCR revealed that Lin28 mRNA expression levels were increased in Lin28A over-expressing cells, $n=3$ (a). Conversely, Let $7 \mathrm{a}, \mathrm{c}$ and $\mathrm{g}$ miRNA expression levels were decreased in Lin28 over-expressing cells, $n=3$ (b). Western blot analysis (c) confirmed that Lin28 protein levels were also increased in over-expressing HEK cells (d), $n=4$. GAPDH and U6 were used as housekeeping genes for mRNA and miRNA expression, respectively. Student's unpaired t-tests were used to compare between experimental groups. Data are represented as mean \pm SEM. HEK human embryonic kidney, miRNA microRNA 


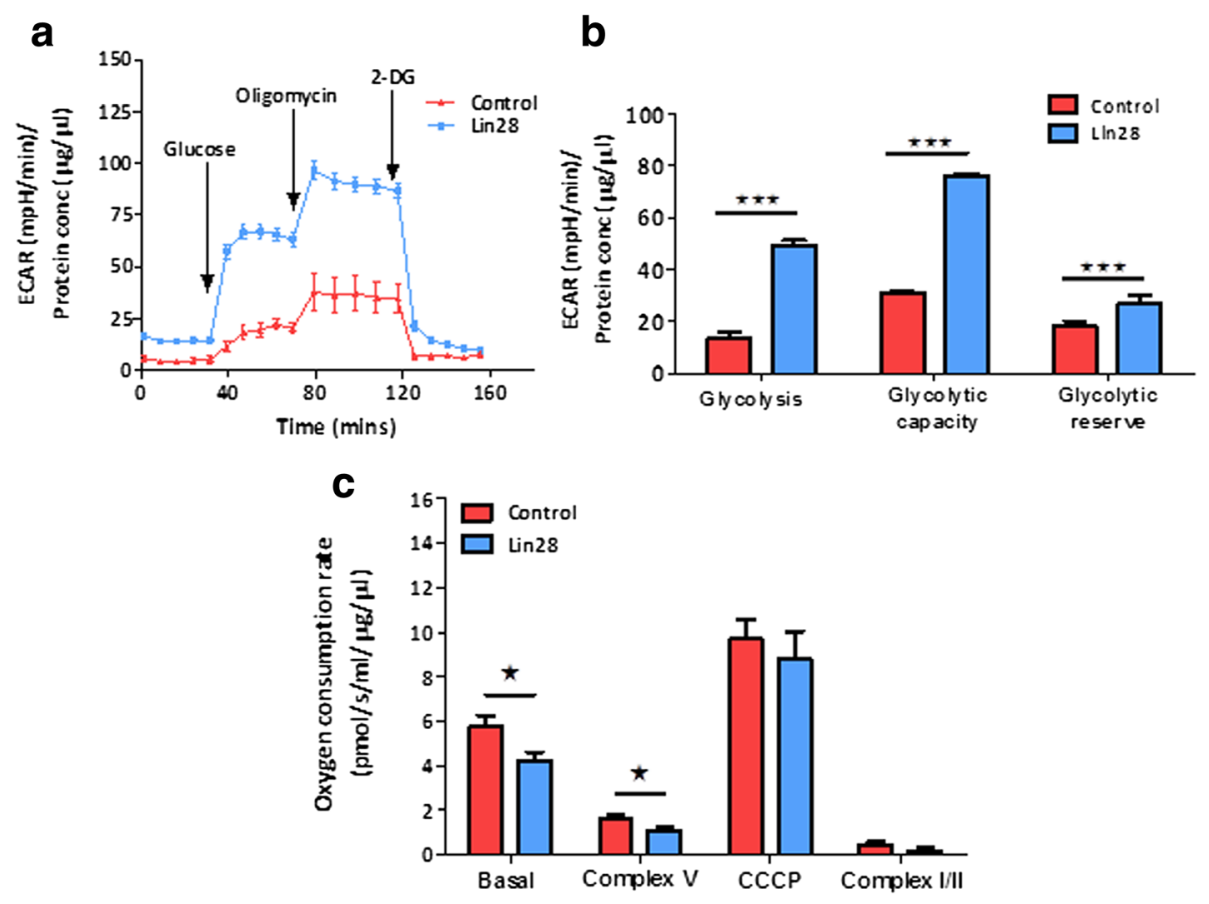

Fig. 2 Lin28A promotes energetic switching to a more glycolytic phenotype. Extracellular flux analysis using the Seahorse XF24 bio-analyser demonstrated that ECAR was significantly increased in Lin28A over-expressing cells after addition of $10 \mathrm{mM}$ glucose (glycolysis). ECAR was also increased after subsequent addition of $1 \mu \mathrm{M}$ oligomycin (glycolytic capacity) and $100 \mathrm{mM}$ 2-DG (glycolytic reserve) (a and b). Conversely, high resolution oxygen respirometry showed that even without stimulation basal oxygen consumption was reduced in over-expressing cells. No changes in maximal respiration (after addition of $1 \mu \mathrm{M}$ CCCP) or spare capacity (after addition of $1 \mu \mathrm{M}$ antimycin and $1 \mu \mathrm{M}$ rotenone) were observed (c). Student's unpaired t-tests were used to compare between experimental groups. Data are represented as mean $\pm S E M, n=5$ for all data points. ECAR extracellular acidification rate, 2-DG 2-deoxyglucose, CCCP carbonyl cyanide m-chlorophenyl hydrazone

reserve $(\mathrm{P}<0.001$, Fig. $2 \mathrm{~b})$ after stimulation with glucose and inhibiting oxidative phosphorylation. However, basal oxidative metabolism, measured by oxygen flux (OCR) (P $<0.05)$ was significantly reduced in Lin28 over-expressers compared to the un-transfected HEK293 cells (Fig. 2c). Interestingly, no significant difference in maximal respiration was observed between the two cell types after respiratory uncoupling of the mitochondrial membrane potential $(\triangle \Psi \mathrm{m})$ with carbonyl cyanide $m$-chlorophenyl hydrazone CCCP $(1 \mu \mathrm{g} / \mathrm{ml})$.

\section{Lin28 over-expression increases cellular proliferation and glucose uptake}

In order to assess the functional consequences of Lin28 over-expression, cellular proliferation and glucose transport were assessed. Increased proliferation of cells overexpressing Lin28A was observed at 72 hours $(\mathrm{P}<0.05)$ and 96 hours $(\mathrm{P}<0.001$, Fig. 3a). 2-Deoxyglucose uptake was also demonstrated to be significantly increased in cells over-expressing Lin28 ( $\mathrm{P}<0.01$, Fig. $3 \mathrm{~b})$.

\section{Lin28 alters several glycolytic enzymes}

In assessing the mechanism of Lin28A's effects in metabolic switching we examined several key enzymes involved in the glycolytic pathway using Western blotting. Hexokinase II (Hex II) was significantly increased in Lin28 over-expressing cells $(\mathrm{P}<0.001$, Fig. $4 \mathrm{c})$ as was pyruvate dehydrogenase ( $\mathrm{PDH}, \mathrm{P}<0.001$, Fig. 4f). Pyruvate kinase muscle isoform 2 (PKM2) was significantly decreased in Lin28A over-expressers $(\mathrm{P}<0.01)$. Lactate dehydrogenase A (LDHA) was also significantly decreased in Lin 28 cells $(\mathrm{P}<0.01$, Fig. $4 \mathrm{~g})$. No differences were observed in hexokinase I (Hex I), pyruvate fructose kinase phosphate (PFKP) compared to age and passage matched controls.

\section{Complex IV is reduced in Lin28A over-expressing cells}

The mitochondrial respiratory complex antibody cocktail (Abcam ab110413) was used to assess any differences in the protein levels of individual respiratory complexes and normalised to nuclear encoded citrate synthase (CS) or manganese superoxide dismutase (MnSOD). In Lin28A over-expressing cells complex IV was significantly decreased $(\mathrm{P}<0.05$, Fig. $5 \mathrm{e})$; however, complex III was significantly increased in the over expressing cells $(\mathrm{P}<0.001)$. No differences were observed in complexes I, II and V when compared to age matched controls. 


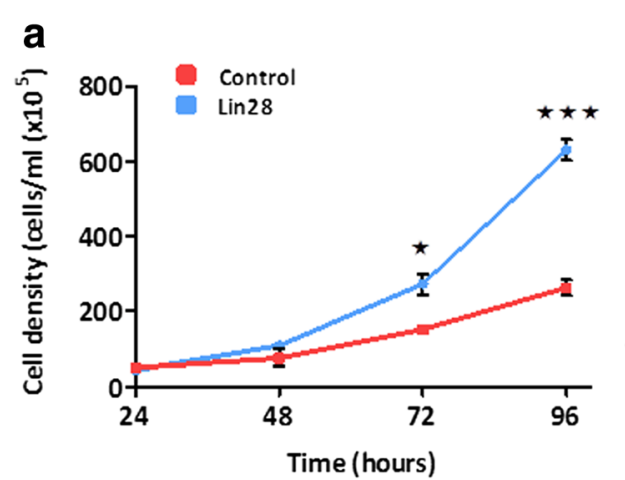

b

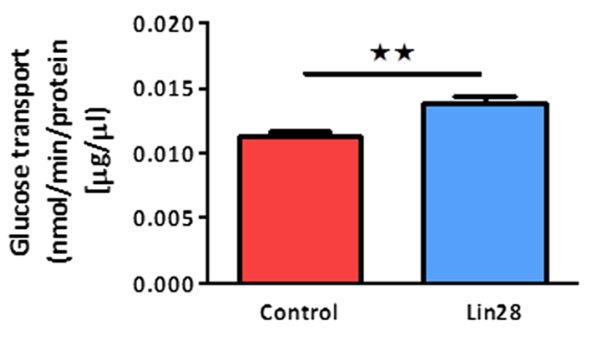

Fig. 3 Lin28A increases cellular proliferation and glucose transport in HEK 293 cells. Proliferation studies revealed that cell density was significantly increased at 72 and 96 hour time points in Lin28A over-expressing cells compared to non-transfected (blank) HEK cells, $\mathrm{n}=3$ (a). Radio-labelled glucose transport assay revealed that 2-deoxyglucose transport was also significantly increased in the Lin28A over-expressing cells $(P<0.01, n=6)(\mathbf{b})$. Student's unpaired t-tests were used to compare between experimental groups. Data are represented as mean \pm SEM. HEK human embryonic kidney

\section{Lin28A also has specific transcriptional effects}

Several markers known to be involved in glycolytic switching were also assessed at the transcriptional level using real-time quantitative PCR. Similar to protein expression, Hex II mRNA was significantly increased in Lin28A over expressing cells $(P>0.01$, Fig. 6a); however, no differences in pyruvate dehydrogenase kinase 2 (PDK2) or hypoxia inducible factor $1 \alpha$ (HIF1 $\alpha)$ mRNA expression was observed (Fig. $6 \mathrm{~b}$ and c, respectively). No significant difference was observed in the nuclear encoded mitochondrial marker citrate synthase (CS) between cell types. Interestingly, the mitophagy markers phosphatase and tensin homolog induced putative kinase 1 (PINK1) and mitofusin 2 (MFN2) were significantly increased in Lin28A over-expressing cells $(\mathrm{P}<0.001$, Fig. $6 \mathrm{e}$ and $\mathrm{P}<0.05$, Fig. 6f, respectively).

\section{Discussion}

In the present study we have identified the distinct metabolic changes occurring in the reprogramming of a transgenic human kidney line using over-expression of Lin28A and concomitant down regulated expression of Let7-g. Notably, this included a metabolic 'switching' from an energetic state relying predominantly on oxidative phosphorylation to a more glycolytic phenotype, whilst aerobic culture conditions remained constant. Indeed, this 'metabolic reprogramming' to a more glycolytic phenotype has been described in detail in previous studies $[1,5,13]$. However, we have outlined several new mechanisms not previously identified that may contribute to Lin28's capacity to increase glycolysis and enhance a cell's metabolic and mitotic performance. We have demonstrated an increase in Hex II at both the transcriptional and protein levels as well as an increase in glucose transport. These effects demonstrate that Lin28's ability to increase Hex II is a crucial step in glycolytic switching. Hex II is the most active isoform of the hexokinase family [17] and increased intracellular Hex II activity is regarded as the rate limiting step in maintaining the glucose concentration gradient by reducing intracellular glucose through phosphorylation of glucose to glucose-6-phosphate [18]. In these cells Hex II appears the key driver of glucose transport which is confirmed by a combination of phosphorylation and increased glucose uptake, which is predicted to occur through the glucose transporters, such as GLUT-1, -2 and GLUT-4 $[5,18-20]$, as well as the sodium glucose co-transporter 2 (SGLT-2) [21]. Interestingly, an increase in PDH and a decrease in LDHA in Lin28A cells confirmed a preference for pyruvate decarboxylation of pyruvate to produce acetyl-CoA rather than conversion of pyruvate to lactate. This reduction in LDHA would further increase the amount of pyruvate for conversion to acetyl-CoA. Of course, acetyl-CoA can be used a substrate in oxidative phosphorylation and may be a compensatory response to an increase in glycolytic rate. A reduction in LDHA is also intriguing because increased LDHA is known to be a driver of glycolysis and subsequently cancer progression, a characteristic previously attributed to Lin28 over expression [22]. Interestingly, PKM2 protein expression was decreased in Lin28A over-expressing cells. This was surprising as much work has outlined the role of PKM2 as a molecular driver for glycolysis in many cancers $[23,24]$. Indeed, results from this study suggest that, at least in HEK cells, PKM2 does not contribute significantly to glycolysis. Indeed, in our model we did not investigate the nature of the specific isoforms of PKM. These couldhave significant consequences for glycolytic metobolism [25], certainly this would require closer scrutiny in relation to Lin28 expression. HIF $1 \alpha$ is also known to be important in the progression of several cancers, including renal cell carcinoma; therefore, HIF1 $\alpha$ 
a

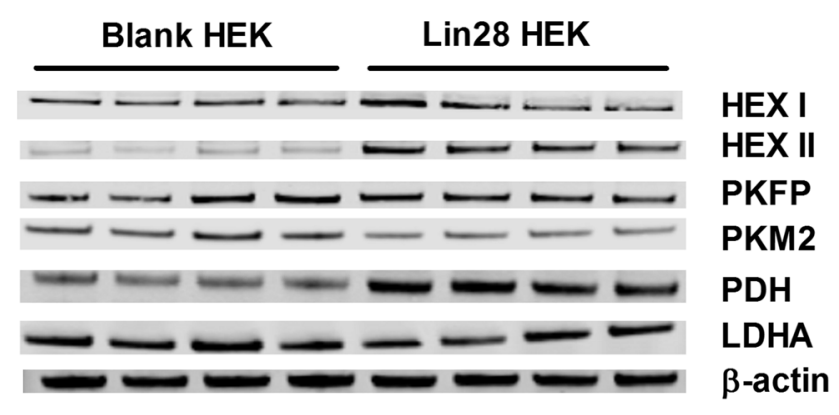

b

HEXI

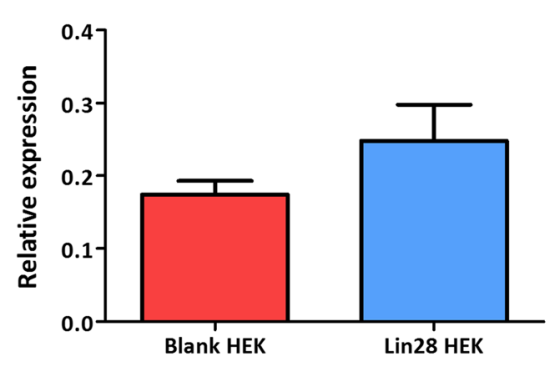

d

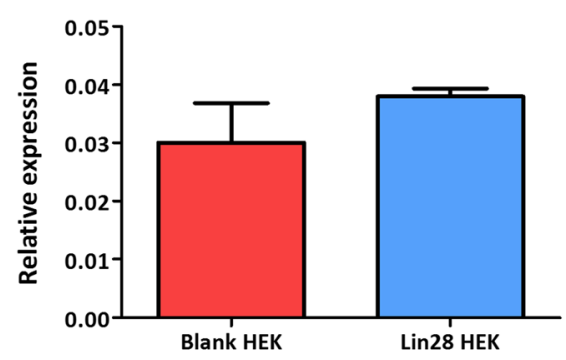

f

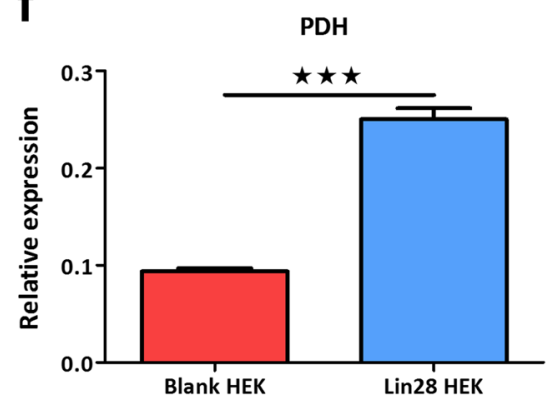

C

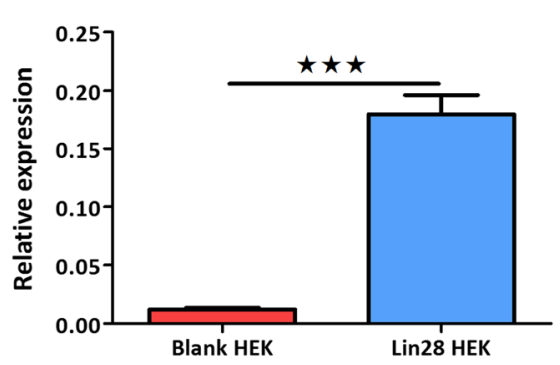

e

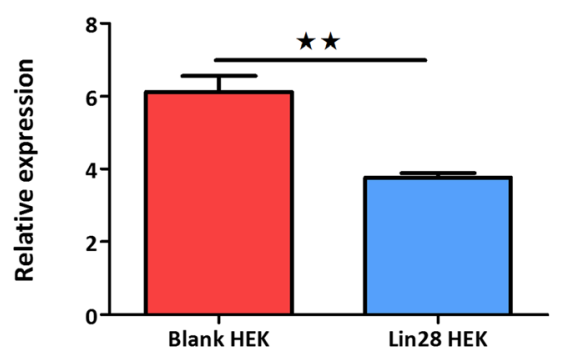

g

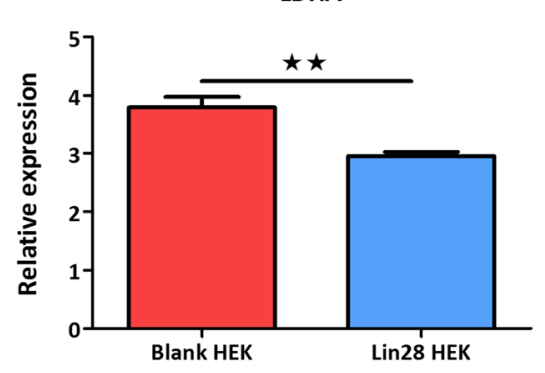

Fig. 4 Lin28A over-expression alters several glycolytic enzymes. Densitometry analysis of Western blot data (a) showed that hexokinase II (c) and PDH (f) expression was significantly increased in Lin28A over-expressers. However, PKM2 (e) and LDHA (g) were significantly decreased in Lin28A over expressers. No change was observed in Hex I (b) or PFKP (d). (Student's unpaired t-tests were used to compare between experimental groups. Data are represented as mean $\pm \mathrm{SEM}, \mathrm{n}=4$. $P D H$ pyruvate dehydrogenase, $P K M 2$ pyruvate kinase muscle isoform 2, LDHA Lactate dehydrogenase $\mathrm{A}$, Hex I hexokinase I, PFKP pyruvate fructose kinase phosphate

expression was assessed [5, 26]. Results from this study showed that Lin28's increase in glycolysis did not appear to be HIF $1 \alpha$-dependent and is consistent with previously published data [5]. Equally, the oncogene c-myc is a known driver of Lin28, but was not overexpressed in control cell lines or in response to Lin28 (Additional file 1: Figure S1). This, combined with a notable reduction in other known cancer-related proteins such as PDK and LDHA, suggests Lin28's role as an oncogene itself may be overstated and may be amenable as an in vitro therapeutic 


\section{a}

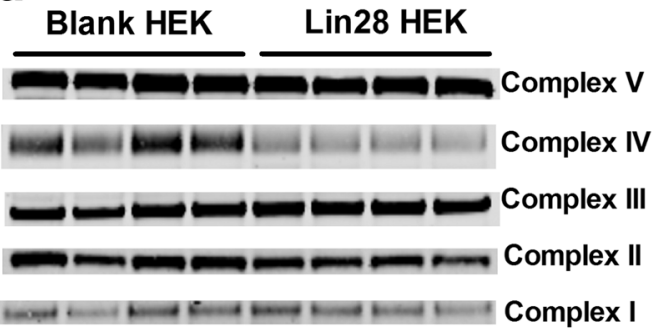

C

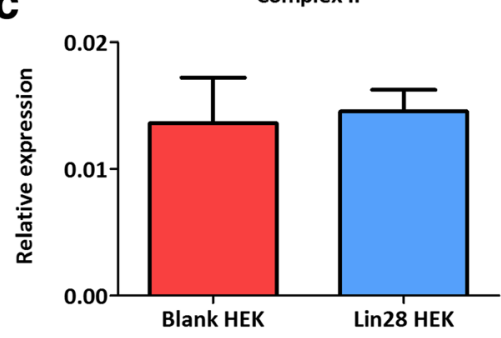

$\mathbf{e}$

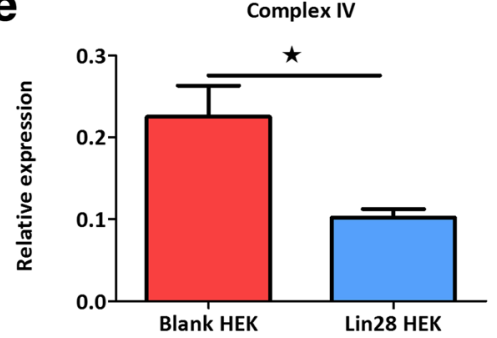

b

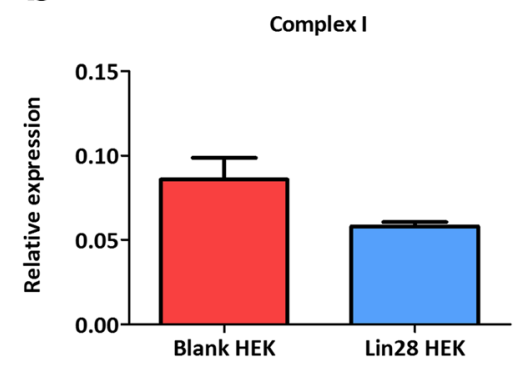

d

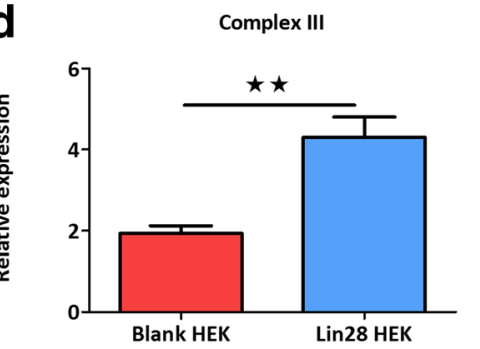

$\mathbf{f}$

Complex V

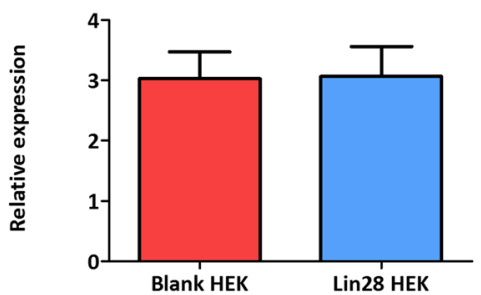

Fig. 5 Lin28A over-expression has specific effects on mitochondrial complexes III and IV. Densitometric analysis of Western blot data (a) revealed no difference in complex I (b), II (c) or Complex V (f) but mitochondrial complex III significantly increased in Lin28A cells (d). A significant reduction in complex IV protein expression was also observed (e). Student's unpaired t-tests were used to compare between experimental groups. Data are represented as mean $\pm S E M, n=4$

target in certain circumstances. Indeed, the mechanism of how up-regulation of stem cell factors, such as Lin28 effectors, can ameliorate the reliance on ox-phos through elegant Let7 anti-miR work corroborates this link [1].

Building on these findings we now demonstrated mRNA transcripts of several mitochondrial-related proteins have yielded additional intriguing results. The mitophagy marker PINK1 (PTEN-induced kinase1) [27] and its downstream effector Mitofusin 2 (MFN2) were significantly increased in Lin28 over expressing cells. This suggests a novel role for Lin28 in promoting energetic switching to a more glycolytic phenotype through mediating enhanced mitochondrial recycling which may underpin the specific effects of Lin28 on oxidative phosphorylation that include a decrease in basal oxygen consumption using a high resolution oxygen respirometry. Further analysis revealed a reduction in the oxygen consuming complex IV proteins which may account for the reduction in basal oxygen consumption and adding further weight to the notion that reduced oxidative metabolism in these cells is driven by Lin28. A reduction in oxygen consumption has been noted in various Lin28 studies utilising cancer cell lines, such as Hep3B cells (Ma et al., 2014). However, in contrast, mouse embryonic fibroblasts (MEF) isolated from mice where Lin28 was over-expressed showed an increase in oxygen consumption rate [13], suggesting Lin28A over expression may have distinct effects depending on cell type $[6,7$, 13]. The remarkable metabolic plasticity we show here suggests that use of synthetic targeted nucleases, such as inducible clustered regularly interspaced short palindromic repeats (CRISPR), or age related inducible expression vectors, may eventually be able to augment favourable changes in cells and tissues of choice.

\section{Conclusions}

Results from this study confirm that augmenting Lin28A expression in differentiated epithelial lineages has the 

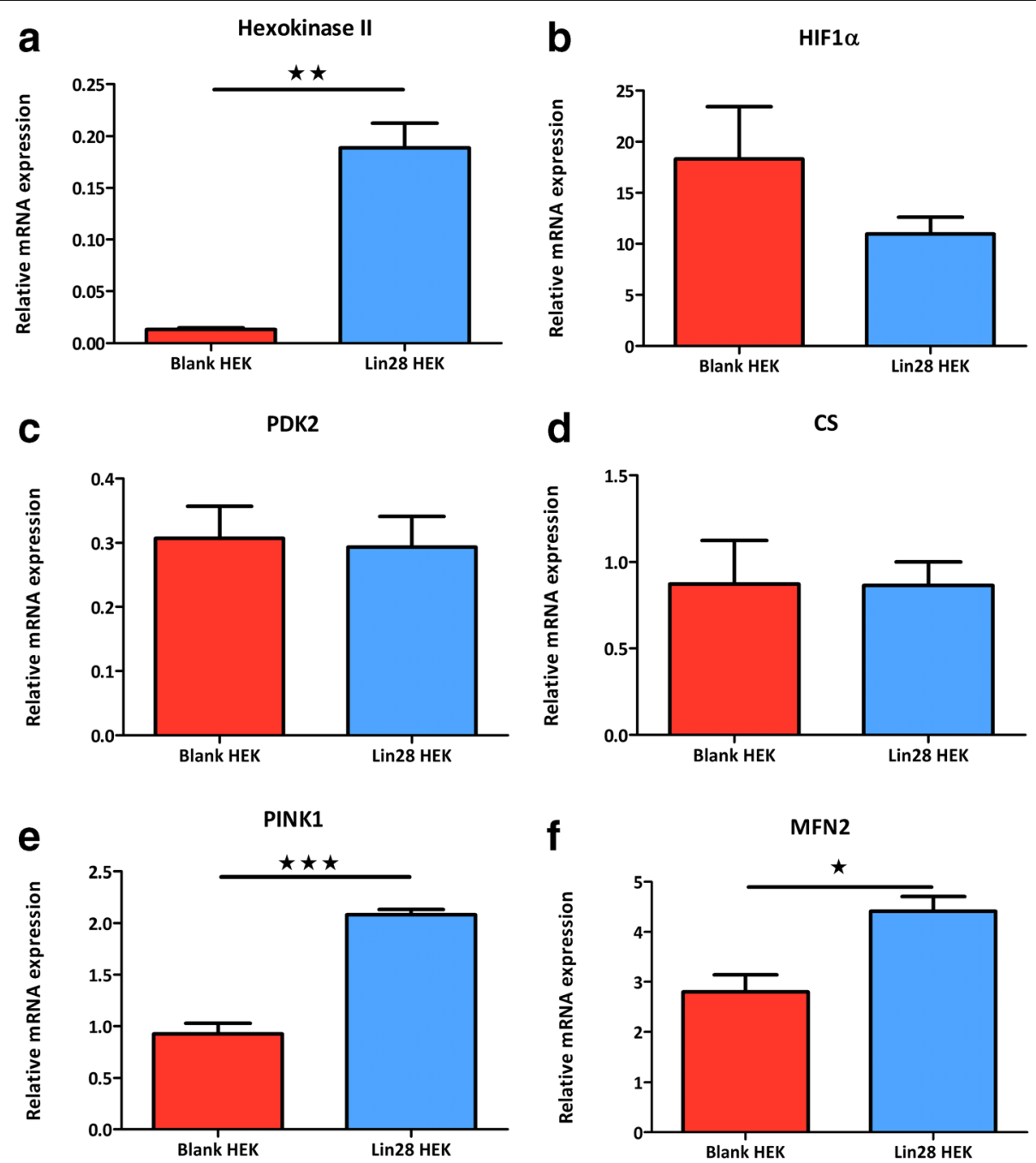

Fig. 6 Lin28A over-expression has various effects on mRNA transcription. Real-time PCR analysis revealed over-expression of Lin28A increased hexokinase II mRNA expression (a). No significant differences were observed in expression levels of HIF1a (b), PDK2 (c) or citrate synthase (d). Mitophagy markers PINK1 and MFN2 were significantly increased in Lin28A over-expressing cells (e and f). 18S was used as a housekeeper gene for normalisation purposes. Student's unpaired t-tests were used to compare between experimental groups. Data are represented as mean \pm SEM, $\mathrm{n}=3$ for each group. HIF1a hypoxia inducible factor $1 \mathrm{a}$, PDK2 pyruvate dehydrogenase kinase 2, PINK1 PTEN-induced kinase1, MFN2 mitofusin 2, CS citrate synthase

potential to reprogram cellular energetics through increasing Hex II expression and activity. A number of degenerative pathologies could be potential beneficiaries of this cellular reprogramming [28-30].

\section{Consent for publishing}

All authors offer their full consent in the publishing of this manuscript. Consent forms are available on request.

\section{Availability of data}

The University of Glasgow, our approved data repository, provides a comprehensive data management and freely available service which supports the principles of open access details of which can be found here: http://www.gla.ac.uk/services/datamanagement/looking afteryourdata/preservation/repositories/.

\section{Additional file}

Additional file 1: Supplementary information. (DOC $164 \mathrm{~kb})$

\section{Abbreviations}

anti-miR: anti microRNA; CCCP: carbonyl cyanide $m$-chlorophenyl hydrazone; CRISPR: clustered regularly interspaced short palindromic repeats; HEK: human embryonic kidney; HEX II: hexokinase 2; LDHA: lactate dehydrogenase; Let-7: lethal-7 family of regulatory; MEF: mouse embryonic fibroblasts; MFN2: mitofusin 2; miRNA: microRNAs; PDH: pyruvate dehydrogenase; PINK1: PTEN-induced kinase1; SGLT-2: sodium glucose co-transporter. 


\section{Competing interests}

The authors declare that they have no competing interests.

\section{Authors' contributions}

CKD: Conception and design, collection and assembly of data, data analysis and interpretation, manuscript writing. IPS: Collection of data, data analysis and interpretation, manuscript writing. JRM: Primary conception and design, data analysis and interpretation, manuscript writing. All authors read and approved the final manuscript.

\section{Funding}

This study was supported by the University of Glasgow Strategic Grant 146123-001

Received: 15 January 2016 Revised: 6 April 2016

Accepted: 11 April 2016 Published online: 26 May 2016

\section{References}

1. Shyh-Chang N, Daley GQ. Lin28: primal regulator of growth and metabolism in stem cells. Cell Stem Cell. 2013;12:395-406.

2. Ahlqvist KJ, Suomalainen A, Hamalainen RH. Stem cells, mitochondria and aging. Biochim Biophys Acta. 1847;2015:1380-6.

3. Poulose N, Raju R. Aging and injury: alterations in cellular energetics and organ function. Aging Dis. 2014;5:101-8.

4. Riera CE, Dillin A. Tipping the metabolic scales towards increased longevity in mammals. Nat Cell Biol. 2015;17:196-203.

5. Ma X et al. Lin28/let-7 axis regulates aerobic glycolysis and cancer progression via PDK1. Nat Commun. 2014;5:5212.

6. Lee TH et al. Functional recapitulation of smooth muscle cells via induced pluripotent stem cells from human aortic smooth muscle cells. Circ Res. 2010;106:120-8.

7. Park JT et al. Repression of let-7 by transforming growth factor-beta1-induced Lin28 upregulates collagen expression in glomerular mesangial cells under diabetic conditions. Am J Physiol Renal Physiol. 2014;307:F1390-1403.

8. Copley MR et al. The Lin28b-let-7-Hmga2 axis determines the higher self-renewal potential of fetal haematopoietic stem cells. Nat Cell Biol. 2013;15:916-25

9. $\mathrm{Yu}$ J et al. Induced pluripotent stem cell lines derived from human somatic cells. Science. 2007:318:1917-20.

10. Viswanathan SR, Daley GQ. Lin28: a microRNA regulator with a macro role. Cell. 2010;140:445-9.

11. Viswanathan SR, Daley GQ, Gregory RI. Selective blockade of microRNA processing by Lin28. Science. 2008;320:97-100.

12. Zhu $\mathrm{H}$ et al. The Lin28/let-7 axis regulates glucose metabolism. Cell. 2011:147:81-94.

13. Shyh-Chang $\mathrm{N}$ et al. Lin28 enhances tissue repair by reprogramming cellular metabolism. Cell. 2013:155:778-92.

14. Zhou J, Ng SB, Chng WJ. LIN28/LIN28B: an emerging oncogenic driver in cancer stem cells. Int J Biochem Cell Biol. 2013:45:973-8.

15. Xu B, Zhang K, Huang Y. Lin28 modulates cell growth and associates with a subset of cell cycle regulator mRNAs in mouse embryonic stem cells. RNA. 2009:15:357-61.

16. Peng $S$ et al. Genome-wide studies reveal that Lin28 enhances the translation of genes important for growth and survival of human embryonic stem cells. Stem Cells. 2011;29:496-504.

17. Mathupala SP, Ko YH, Pedersen PL. Hexokinase II: cancer's double-edged sword acting as both facilitator and gatekeeper of malignancy when bound to mitochondria. Oncogene. 2006:25:4777-86.

18. Burcelin $\mathrm{R}$ et al. Regulation of glucose transporter and hexokinase II expression in tissues of diabetic rats. Am J Physiol. 1993:265:E392-401.

19. Liu F et al. Development of a novel GLUT4 translocation assay for identifying potential novel therapeutic targets for insulin sensitization. Biochem J. 2009:418:413-20.

20. Thorens B, Mueckler M. Glucose transporters in the 21st Century. Am J Physiol Endocrinol Metab. 2010:298:E141-145.

21. Han $Y$ et al. SGLT inhibitors attenuate NO-dependent vascular relaxation in mouse pulmonary artery. FASEB J. 2014:28:677.3.

22. Allison $\mathrm{SJ}$ et al. Identification of $\mathrm{LDH}-\mathrm{A}$ as a therapeutic target for cancer cell killing via (i) p53/NAD(H)-dependent and (ii) p53-independent pathways. Oncogenesis. 2014;3:e102.
23. Wong N, Ojo D, Yan J, Tang D. PKM2 contributes to cancer metabolism. Cancer Lett. 2015:356:184-91.

24. Wu S, Le H. Dual roles of PKM2 in cancer metabolism. Acta Biochim Biophys Sin (Shanghai). 2013:45:27-35.

25. Israelsen WJ et al. PKM2 isoform-specific deletion reveals a differential requirement for pyruvate kinase in tumor cells. Cell. 2013;155:397-409.

26. Kondo K, Klco J, Nakamura E, Lechpammer M, Kaelin Jr WG. Inhibition of HIF is necessary for tumor suppression by the von Hippel-Lindau protein. Cancer Cell. 2002:1:237-46.

27. Patel AS et al. Epithelial cell mitochondrial dysfunction and PINK1 are induced by transforming growth factor-beta1 in pulmonary fibrosis. PLoS One. 2015;10:e0121246.

28. Yu E et al. Mitochondrial DNA damage can promote atherosclerosis independently of reactive oxygen species through effects on smooth muscle cells and monocytes and correlates with higher-risk plaques in humans. Circulation. 2013;128:702-12.

29. Pils A, Winklhofer KF. Parkin, PINK1 and mitochondrial integrity: emerging concepts of mitochondrial dysfunction in Parkinson's disease. Acta Neuropathol. 2012;123:173-88.

30. Semi K, Matsuda Y, Ohnishi K, Yamada Y. Cellular reprogramming and cancer development. Int J Cancer. 2013;132:1240-8.

\section{Submit your next manuscript to BioMed Central and we will help you at every step:}

- We accept pre-submission inquiries

- Our selector tool helps you to find the most relevant journal

- We provide round the clock customer support

- Convenient online submission

- Thorough peer review

- Inclusion in PubMed and all major indexing services

- Maximum visibility for your research

Submit your manuscript at www.biomedcentral.com/submit 